Original Article

\title{
Effects of controlled condylar rotation exercise on symmetrical mouth opening in patients with temporomandibular disorder
}

\author{
Jae-Seop Oh, PT, PhD ${ }^{1)}$, Si-Hyun Kim, PT, PhD ${ }^{2)}$, Kyue-Nam Park, PT, PhD ${ }^{3)^{*}}$ \\ 1) Department of Physical Therapy, College of Biomedical Science and Engineering, Inje University, \\ Republic of Korea \\ 2) Kinetic Ergocise Based on Movement Analysis Laboratory, Republic of Korea \\ 3) Department of Physical Therapy, College of Medical Science, Jeonju University: 303 Cheonjam-ro, \\ Wansan-gu, Jeonju-si, Jeollabuk-do, Republic of Korea
}

\begin{abstract}
Purpose] The purpose of this study was to determine the effects of a 4-week program of active condylar rotation exercise on the extent of the lateral mandibular shift during mouth opening in patients with temporomandibular disorder. [Subjects and Methods] Patients with temporomandibular disorder $(\mathrm{n}=12 ; 7$ men and 5 women) were recruited. The active range of temporomandibular motion was recorded using 3-dimensional ultrasound-based motion analysis. The paired t-test was used to assess changes in lateral mandibular shift before and after active condylar rotation exercise. [Results] The degree of the lateral mandibular shift during mouth opening and the mouth opening-lateral mandibular shift ratio were significantly lower after active condylar rotation exercise than before the exercise. [Conclusion] Active condylar rotation exercise may effectively reduce the degree of the lateral mandibular shift during mouth opening to produce symmetrical mouth opening in patients with mild temporomandibular disorder.
\end{abstract}

Key words: Active condylar rotation exercise, Lateral mandibular shift, Temporomandibular disorder

(This article was submitted Nov. 25, 2014, and was accepted Jan. 11, 2015)

\section{INTRODUCTION}

Temporomandibular joint (TMJ) disorder (TMD) is defined as pain in the masticatory muscles. Some patients occasionally experience severe pain while chewing ${ }^{1)}$. The characteristics of TMD are orofacial pain, restricted range of TMJ motion, joint clicking, and headaches ${ }^{1)}$. A lateral mandibular shift during mouth opening indicates persistent TMD, which is an important factor for evaluating and managing patients with TMJ problems ${ }^{2-4)}$. A C-shaped lateral mandibular shift during mouth opening is observed in individuals with TMJ impairment ${ }^{5)}$, whereas an S-shaped lateral mandibular shift is observed in patients with TMJ subluxation ${ }^{6}$.

The early phase of mouth opening, which is defined as the first $35-50 \%$ of the mouth opening range, primarily involves a rolling motion of the $\mathrm{TMJ}^{7}, 8$ ). The late phase involves a gradual transition from a primarily posterior rolling motion to anterior translation ${ }^{9)}$. Thus, translation motion of the TMJ is required for maximal mouth opening. However, an early

*Corresponding author. Kyue-Nam Park (E-mail: knpark@ jj.ac.kr)

(C2015 The Society of Physical Therapy Science. Published by IPEC Inc. This is an open-access article distributed under the terms of the Creative Commons Attribution Non-Commercial No Derivatives (by-ncnd) License $<$ http://creativecommons.org/licenses/by-nc-nd/3.0/>. and excessive lateral mandibular shift during maximal mouth opening induces asymmetry. A previous study suggested that maintaining a resting tongue position before mouth opening can reduce this asymmetry ${ }^{10)}$. A resting tongue position is achieved when the tip of the tongue is placed on the hard palate, behind the front teeth ${ }^{10)}$. A previous case study demonstrated the effects of a 6-week neuromuscular control exercise program, including a resting tongue position and an automobilization technique, on TMJ locking and pain. Another study demonstrated that 6 weeks of physical therapy in addition to active condylar rotation exercise (ACRE) using the tongue effectively reduces TMD symptoms ${ }^{11)}$.

Although the resting tongue position is useful for determining the midline position of the jaw, no study has investigated the effects of ACRE alone on symmetrical mouth opening while maintaining a resting tongue position. Here, we investigated the effects of a 4-week ACRE intervention on the reduction of lateral mandibular shift during mouth opening and the ratio of lateral mandibular shift to maximal mouth opening in patients with TMD.

\section{SUBJECTS AND METHODS}

We recruited 28 participants with TMD from the INJE University in Korea. Sixteen participants who did not meet the inclusion criteria were excluded from this study. The remaining 12 participants were 7 men and 5 women, with a mean age of $20.08 \pm 1.62$ years, mean weight of $62.83 \pm$ 
$10.94 \mathrm{~kg}$, and mean height of $171.61 \pm 7.55 \mathrm{~cm}$. The criteria in Axis 1 of the Research Diagnostic Criteria for TMD (RDC/TMD-Axis1) were used as the inclusion criteria; all patients were assessed based on these criteria by Examiner $1^{12)}$. All study participants were classified as having RDC/ TMD-Axis1 Group Ib diagnoses based on the diagnostic criteria. The exclusion criteria were (1) symptoms such as migraine, intracranial neoplasm, toothache, and neuralgia; (2) systemic disease; (3) history of surgical treatment for TMD; (4) medication intake in the $72 \mathrm{~h}$ before measurement; and (5) history of participation in physical therapy and exercise in a clinic ${ }^{13)}$. All the patients signed an informed consent form approved by the Inje University Ethics Committee for Human Investigations.

TMJ motion was measured before the 4-week ACRE intervention. A 3-dimensional ultrasonic motion analysis system was used (CMS-HS; Zebris Medizintechnik GmbH, Isny, Germany) for the measurement. The participants were seated looking straight ahead, and their thoracic region was supported by the backrest of the chair. The examiner attached 2 single active markers to the philtrum and midpoint of the lower jaw. The surface markers were fixed using adhesive tape to prevent their movement and resultant motion artifact during mouth opening. The transducer sensor was placed in front of the participant's face. The sampling rate was $60 \mathrm{~Hz}$. Calibration was performed with a resting closed mouth and neutral head position, which was maintained in a vertical upright position without rotation or lateral bending (subjectively determined by each participant) ${ }^{14)}$. The participants were asked to minimize both neck extension and frowning during mouth opening. In each assessment, the participants were asked to maintain a relaxed closed mouth, open the mouth maximally, and then maintain end-range mouth opening for $3 \mathrm{~s}^{15}$. For familiarization before recording, the participants repeated 5 trials of opening-closing movements. After completing the familiarization, the TMJ motion was recorded 3 times. The participants rested for $30 \mathrm{~s}$ between trials to minimize fatigue. Windata 2.22.25 software (Zebris Medizintechnik GmbH, Isny, Germany) was used to calculate the TMJ motion. Data regarding TMJ motion (extent of lateral mandibular shift and maximal mouth opening) were recorded while participants maintained end-range mouth opening for $3 \mathrm{~s}$. TMJ motion was defined as the change in lower jaw motion relative to that in the upper jaw. Maximal mouth opening was calculated as the difference between the upper and lower jaw coordinates in the sagittal plane. The extent of lateral mandibular shift at the end of mouth opening was defined as the difference between the upper and lower jaw coordinates in the frontal plane. To compare the change in lateral mandibular shift during mouth opening, we calculated the ratio of the extent of lateral mandibular shift relative to the extent of mouth opening (lateral mandibular shift ratio $=$ extent of lateral mandibular shift $\div$ extent of mouth opening). The mean values of 3 trials were used to assess TMJ motion. A previous study demonstrated that the intratester reliability intraclass correlation coefficient for TMJ motion using the Zebris motion analysis system was $0.83-0.93^{16)}$. TMJ motion was also measured after the 4-week ACRE intervention. The measurement procedure was the same as that used before the intervention.
A research assistant gave individual instructions to all the participants before starting each exercise. The participants attended the ACRE program for 5 consecutive days each week during a 4-week period in an exercise room. During each exercise period, the participants were asked not to engage in other types of exercise or treatment for TMD. ACRE was implemented as follows. The participants were asked to place the tongue in the resting position. The resting tongue position was achieved by placing the tip of the tongue on the anterior palate, just posterior to the upper front teeth ${ }^{2)}$. With the tongue in the resting position, the participants opened their mouth while trying to maintain the tongue on the anterior palate to avoid anterior condylar translation ${ }^{2)}$. When the participants failed to maintain the tongue in the resting position, they were instructed to stop the mouth opening and return to the starting position to repeat the exercise. Controlled ACRE was repeated 6 times at a frequency of 6 sessions per day ${ }^{2}$.

All data showed a normal distribution according to the Kolmogorov-Smirnov test. Paired t-tests were performed to compare changes in the dependent variables (extent of mouth opening, lateral mandibular shift, and lateral mandibular shift-mouth opening ratio) between, before, and after the ACRE intervention. Data are expressed as mean \pm standard deviation (SD). The level of statistical significance was set at $\mathrm{p}<0.05$. Statistical analyses were performed using SPSS ver. 20.0 software (SPSS Inc., Chicago, IL, USA).

\section{RESULTS}

The extent of lateral mandibular shift was significantly lower after the intervention $(2.03 \pm 0.64 \mathrm{~mm})$ than before the intervention $(4.00 \pm 1.31 \mathrm{~mm})(\mathrm{p}<0.05)$. The lateral mandibular shift-mouth opening ratio was significantly lower after the intervention $(7.20 \pm 2.88 \%)$ than before the intervention $(9.33 \pm 2.80 \%)(\mathrm{p}<0.05)$.

\section{DISCUSSION}

Lateral mandibular shift during mouth opening indicates the possibility of persistent $\mathrm{TMD}^{2-4)}$. However, there is currently a lack of evidence to support the effects of therapeutic exercise alone on the reduction of lateral mandibular shift in the management of TMD. To date, this is the first demonstration of the effects of a 4-week ACRE program on the reduction of lateral mandibular shift upon mouth opening in patients with TMD.

The lateral mandibular shift-mouth opening ratio after the intervention was significantly lower than that before the intervention. This demonstrates the effectiveness of ACRE on reducing the lateral mandibular shift and improving symmetry during mouth opening. Consistent with our results, a case report suggested that short-term neuromuscular control exercise can help to reduce the $\mathrm{C}$-curved lateral mandibular shift during mouth opening, although an automobilization technique was applied with the exercise ${ }^{2)}$. Lateral deviation during mouth opening may be caused by early translation of the $\mathrm{TMJ}^{2}$. The ACRE in our study focused on rolling arthrokinematics and prevention of early translation of the TMJ during mouth opening. The participants in this study may 
have benefited from an exercise to address the rolling movement in the TMJ, resulting in reduction of lateral deviation during mouth opening.

The tongue was positioned below the anterior palate during the ACRE to avoid anterior condylar translation and to prevent early contraction of the opposite side of the lateral pterygoid ${ }^{4}$. The resting tongue position before mouth opening helps to maintain the midline position of the jaw ${ }^{10)}$. Maintenance of the resting tongue position may help to keep the midline position of the jaw stable during mouth opening. This postural education using ACRE may have contributed to the decreased lateral mandibular shift after the 4-week intervention.

One of the limitations of this study is that all the participants with TMD had mild symptoms. Patients with severe symptoms should be evaluated in future studies. Additional studies are also needed to compare the effects of ACRE and other coordination exercises for symmetrical mouth opening, such as exercises that utilize visual feedback with a mirror, or tactile feedback using a finger to palpate the lateral pole of the mandibular condyle bilaterally in patients with $\mathrm{TMD}^{10)}$.

In conclusion, a 4-week ACRE intervention effectively reduced the lateral mandibular shift during mouth opening. This study demonstrated the importance of ACRE with the tongue in the resting position in the reduction of lateral mandibular shift during mouth opening. ACRE may help to improve symmetrical mouth opening and mandibular neuromuscular control in individuals with TMD characterized by a lateral mandibular shift.

\section{REFERENCES}

1) Ucar M, Sarp Ü, Koca İ, et al.: Effectiveness of a home exercise program in combination with ultrasound therapy for temporomandibular joint disorders. J Phys Ther Sci, 2014, 26: 1847-1849. [Medline] [CrossRef]

2) Hoglund LT, Scott BW: Automobilization intervention and exercise for temporomandibular joint open lock. J Manual Manip Ther, 2012, 20: 182 191. [Medline] [CrossRef]

3) Chossegros C, Cheynet F, Guyot L, et al.: Posterior disk displacement of the TMJ: MRI evidence in two cases. Cranio, 2001, 19: 289-293. [Medline]

4) Dutton M: Orthopaedic Examination, Evaluation, and Intervention, 2nd ed. New York: McGraw-Hill Medical, 2008.

5) CG W: TMJ Disorders: Management of the Craniomandibular Complex, 1st ed. New York: Churchill Livingstone, 1988

6) Wood GD, Branco JA: A comparison of three methods of measuring maximal opening of the mouth. J Oral Surg, 1979, 37: 175-177. [Medline]

7) Yustin DC, Rieger MR, McGuckin RS, et al.: Determination of the existence of hinge movements of the temporomandibular joint during normal opening by Cine-MRI and computer digital addition. J Prosthodont, 1993, 2: 190-195. [Medline] [CrossRef]

8) Rocabado M: Arthrokinematics of the temporomandibular joint. Dent Clin North Am, 1983, 27: 573-594. [Medline]

9) Ferrario VF, Sforza C, Miani A Jr, et al.: Open-close movements in the human temporomandibular joint: does a pure rotation around the intercondylar hinge axis exist? J Oral Rehabil, 1996, 23: 401-408. [Medline] [CrossRef]

10) Kisner C, Colby LA: Therapeutic exercise: foundations and techniques, 6th ed. Philadelphia: F.A. Davis, 2012.

11) Oh DW, Kim KS, Lee GW: The effect of physiotherapy on post-temporomandibular joint surgery patients. J Oral Rehabil, 2002, 29: 441-446. [Medline] [CrossRef]

12) Lauriti L, Silva PF, Politti F, et al.: Pattern of electromyographic activity in mastication muscles of adolescents with temporomandibular disorder. J Phys Ther Sci, 2013, 25: 1303-1307. [Medline] [CrossRef]

13) Galhardo AP, da Costa Leite C, Gebrim EM, et al.: The correlation of research diagnostic criteria for temporomandibular disorders and magnetic resonance imaging: a study of diagnostic accuracy. Oral Surg Oral Med Oral Pathol Oral Radiol, 2013, 115: 277-284. [Medline] [CrossRef]

14) Cagnie B, Cools A, De Loose V, et al.: Reliability and normative database of the Zebris cervical range-of-motion system in healthy controls with preliminary validation in a group of patients with neck pain. J Manipulative Physiol Ther, 2007, 30: 450-455. [Medline] [CrossRef]

15) Baltali E, Zhao KD, Koff MF, et al.: A method for quantifying condylar motion in patients with osteoarthritis using an electromagnetic tracking device and computed tomography imaging. J Oral Maxillofac Surg, 2008, 66: 848-857. [Medline] [CrossRef]

16) Seedorf $\mathrm{H}$, Seetzen F, Scholz A, et al.: Impact of posterior occlusal support on the condylar position. J Oral Rehabil, 2004, 31: 759-763. [Medline] [CrossRef] 